\title{
Correlation Effect Between Transmitter and Receiver Azimuthal Directions on the Illumination Function from a Random Rough Surface
}

\author{
Eric Heitz ${ }^{\mathrm{a} *}$, Christophe Bourlier ${ }^{\mathrm{b}}$ and Nicolas Pinel ${ }^{\mathrm{b}}$ \\ a Inria-Jean Kuntzmann Laboratory (Université de Grenoble and CNRS) \\ 655 avenue de l'Europe, 38334 Saint Ismier Cedex, France \\ b IETR Laboratory - Université de Nantes, Polytech Nantes, Rue Christain Pauc, La \\ Chantrerie, 44306 Nantes Cedex 3, France
}

(Received 24 January 2013)

\begin{abstract}
The resolution of some problems of electromagnetic scattering from random rough surfaces implies the derivation of the illumination function, especially when the geometrical optics approximation is valid. In current models, the shadowing effects occurring for both the transmitter and the receiver are assumed to be independent of their azimuthal directions. This assumption makes it possible to compute separately the shadowing probabilities in each direction. However, if the transmitter and receiver azimuthal directions are close, these probabilities become strongly correlated. In such a configuration, the uncorrelation approximation induces an overestimation of the average illumination function up to $10 \%$ as well as a discontinuity. In this paper, assuming surfaces of isotropic Gaussian statistics with independent heights and slopes, this correlation is taken into account. Comparisons of our model with Monte Carlo simulations are made and show a very good agreement, which validates our approach.
\end{abstract}

\section{Introduction}

Resolving some problems of electromagnetic wave scattering from random rough surfaces needs the derivation of a so-called illumination function, especially when the geometrical optics approximation can be applied. Conventional illumination functions, for example Wagner's [1] and Smith's [2] models, can be dated back to the 1960s, and are widely applied. These models assume one-dimensional (1-D) stationary surfaces of Gaussian statistics for which the heights and the slopes are independent random variables. Brown [3] extended the illumination function to the case of non-Gaussian surfaces with the same height-slope independence assumption. Bourlier et al. [4, 5] investigated this problem and showed, by comparing the Wagner, Smith and Ricciardi-Sato models, that Smith's illumination function was the most accurate and that considering the dependence between the heights and the slopes weakly improves the result. Bourlier et al. [5, 6] also extended the formulation to the bistatic case, for which the transmitter and the receiver are in different directions. The bistatic model consists in multiplying two independent one-dimensional monostatic illumination functions: the first one associated to the transmitter and the second one to the receiver. However, this assumption is not valid for many two-dimensional configurations. Indeed, if the transmitter and receiver azimuthal directions are close, the illumination probabilities associated to

*Corresponding author. Email: eric.heitz@inria.fr 
these directions become strongly correlated and then, they cannot be multiplied. While this case is often neglected, it is not unusual in real-life situations. Typically, in long-range acquisition when the transmitter and the receiver are located on the same device, their directions are close comparatively to the faraway target.

Based on Smith's approach [2], this paper proposes to model this effect. Like in the original Smith's model [2], we assume an isotropic rough surface where the heights and the slopes are independent Gaussian ramdom variables. We propose an extension of Smith's 1-D monostatic illumination function to the 2-D case by accounting for the correlation between the transmitter and receiver azimuthal directions.

Section 2 reviews Smith's monostatic illumination function [2], its extension to the bistatic 2-D case proposed by Bourlier et al. [5, 6], and also discusses of the problem of the correlation between the azimuthal directions. Our model is derived in Section 2, and Section 3 presents some comparisons with a Monte-Carlo process.

Compared to previous work, our contribution can be summarized as the introduction of a correction coefficient $r_{0}$ in Bourlier's model. Our derivation is mathematically sound and based on probability theory. We also propose a simple analytical approximation of this coefficient. Then, the extension of Boulier's model to our model requires a simple evaluation of our approximation of $r_{0}$.

\section{Illumination Function for Gaussian Random Rough Surfaces}

We review the monostatic illumination function for a 1-D Gaussian rough surface proposed by Smith [2] in subsection 2.1, and its extension to a 2-D surface surface and to a bistatic configuration proposed by Bourlier et al. $[5,6]$ in subsection 2.2. Next, we introduce our new bistatic model for a 2-D surface in subsection 2.3 and develop it in subsections 2.3.1, 2.3.2 and 2.3.4.

\subsection{Monostatic Illumination Function for 1-D Surfaces}

Any point $M(\zeta, \gamma)$ of the surface is described by its height $\zeta$ and its slope $\gamma$ (see Fig. 1 with a particular point $M=F)$. The heights and slopes are independent Gaussian random variables defined respectively by $\zeta \sim \mathcal{N}\left(0, \sigma_{\zeta}^{2}\right)$ and $\gamma \sim \mathcal{N}\left(0, \sigma_{\gamma}^{2}\right)$, where $\mathcal{N}(m, \sigma)$ is the normal law of mean value $m$ and of variance $\sigma^{2}$. The associated PDFs (probability density functions) are $p_{\zeta}$ and $p_{\gamma}$, respectively, and the CDFs (cumulative distribution functions) are denoted as $P_{\zeta}$ and $P_{\gamma}$, respectively.

In Fig. 1, the observation (receiver) from direction $A$ has an angle $\theta_{A}$ defined from the vertical $z$ and its associated slope is $\mu_{A}=\cot \theta_{A}$.

The illumination function $S(F, A)$ gives the probability that a surface point $F\left(\zeta_{0}, \gamma_{0}\right)$ with height $\zeta_{0}$ and slope $\gamma_{0}$ is visible from the receiver $A$ and is expressed as the product of two probabilities: $S(F, A)=S_{\gamma}\left(\gamma_{0}, A\right) S_{\zeta}\left(\zeta_{0}, A\right)$. Since our derivation is strongly based on probability theory, we adopt a probability notation and terminology. We call an event a set of outcomes to which a probability is assigned. The notation of the events and the associated probabilities is detailed in Table 1.

The probability $S_{\gamma}\left(\gamma_{0}, A\right)$ that "the point $F$ is not self-shadowed" is the probability that the slope $\gamma_{0}$ of surface the point $F$ is smaller than that of the receiver $\mu_{A}>0$. Thus, this probability is a binary value given by the Heaviside function $\Upsilon$ as

$$
S_{\gamma}\left(\gamma_{0}, A\right)=\Upsilon\left(\mu_{A}-\gamma_{0}\right)
$$




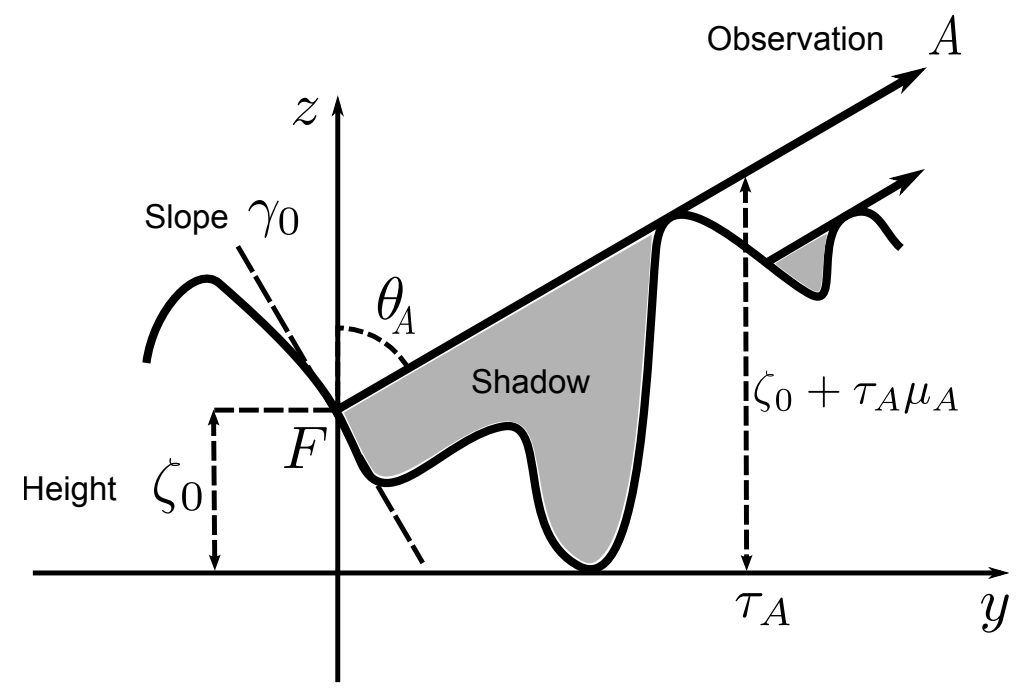

(a)

Figure 1. Illustration of the monostatic illumination function.

\begin{tabular}{cl}
\hline Probability & Associated event \\
\hline$S(F, A)$ & The point $F$ is visible from direction $A$ \\
$S_{\gamma}\left(\gamma_{0}, A\right)$ & The point $F$ is not self-shadowed \\
$S_{\zeta}\left(\zeta_{0}, A\right)$ & The ray " $F A$ " is above the surface at $\left.\tau_{A} \in\right] 0, \infty[$ \\
$S\left(\zeta_{0}, \bar{A}_{\tau_{A}} \mid A_{\tau_{A}-d \tau}\right)$ & $\begin{array}{l}\text { The ray " } F A \text { " intersects the surface at } \tau_{A} \\
\text { given that it is above the surface at } \tau_{A}-d \tau\end{array}$ \\
\hline
\end{tabular}

The probability $S_{\zeta}\left(\zeta_{0}, A\right)$ of the event "the ray " $F A$ " is above the surface at $\left.\tau_{A} \in\right] 0, \infty["$ is

$$
S_{\zeta}\left(\zeta_{0}, A\right)=\exp \left[-\int_{0}^{\infty} S\left(\zeta_{0}, \bar{A}_{\tau_{A}} \mid A_{\tau_{A}-d \tau}\right) d \tau_{A}\right]
$$

where

$$
S\left(\zeta_{0}, \bar{A}_{\tau_{A}} \mid A_{\tau_{A}-d \tau}\right)=\Lambda\left(\mu_{A}\right) \frac{\mu_{A} p_{\zeta}\left(\zeta_{0}+\tau_{A} \mu_{A}\right)}{P_{\zeta}\left(\zeta_{0}+\tau_{A} \mu_{A}\right)}
$$

Note that $S\left(\zeta_{0}, \bar{A}_{\tau_{A}} \mid A_{\tau_{A}-d \tau}\right)$ used to be denoted as $g$ in previous work. We changed it into a probability notation to ensure the consistency throughout the paper. Moreover,

$$
\Lambda\left(\mu_{A}\right)=\frac{\sigma_{\gamma}}{\sqrt{2 \pi} \mu_{A}} \exp \left(-\frac{\mu_{A}^{2}}{2 \sigma_{\gamma}^{2}}\right)-\operatorname{erfc}\left(\frac{\mu_{A}}{\sqrt{2} \sigma_{\gamma}}\right)
$$

and Equation (2) has an analytical expression

$$
\begin{aligned}
S_{\zeta}\left(\zeta_{0}, A\right) & =P_{\zeta}\left(\zeta_{0}\right)^{\Lambda\left(\mu_{A}\right)} \\
& =\left[1-\frac{1}{2} \operatorname{erfc}\left(\frac{\zeta_{0}}{\sqrt{2} \sigma_{\zeta}}\right)\right]^{\Lambda\left(\mu_{A}\right)}
\end{aligned}
$$




\subsection{Bistatic Illumination Function for 2-D Surfaces}

For 2-D surfaces described by $z=\zeta(x, y)$, a surface point has (at least) two slopes: $\gamma_{x}$, the slope with respect to the $x$ direction and $\gamma_{y}$, the slope with respect to the $y$ direction. To simplify the formulation, we consider an isotropic surface, leading to $\left(\gamma_{x}, \gamma_{y}\right) \sim \mathcal{N}\left(0, \sigma_{\gamma}^{2}\right)$. In addition, the receiver is defined from the point $A=\left(\theta_{A}, \phi_{A}\right)$ and the transmitter from the point $B=\left(\theta_{B}, \phi_{B}\right)$ (see Fig. 2). They are defined in spherical coordinates with polar angles $\theta_{A}, \theta_{B} \in[0, \pi / 2]$ and azimuthal angles $\phi_{A}, \phi_{B} \in[0,2 \pi]$. The associated slopes are $\mu_{A}=\cot \theta_{A}$ and $\mu_{B}=\cot \theta_{B}$, respectively. Since we consider an isotropic surface, we define the absolute azimuthal angle difference $\phi=\min \left(\left|\phi_{A}-\phi_{B}\right|,\left|\mu_{A}-\mu_{B}-2 \pi\right|\right)$ and $\phi \in[0, \pi]$.

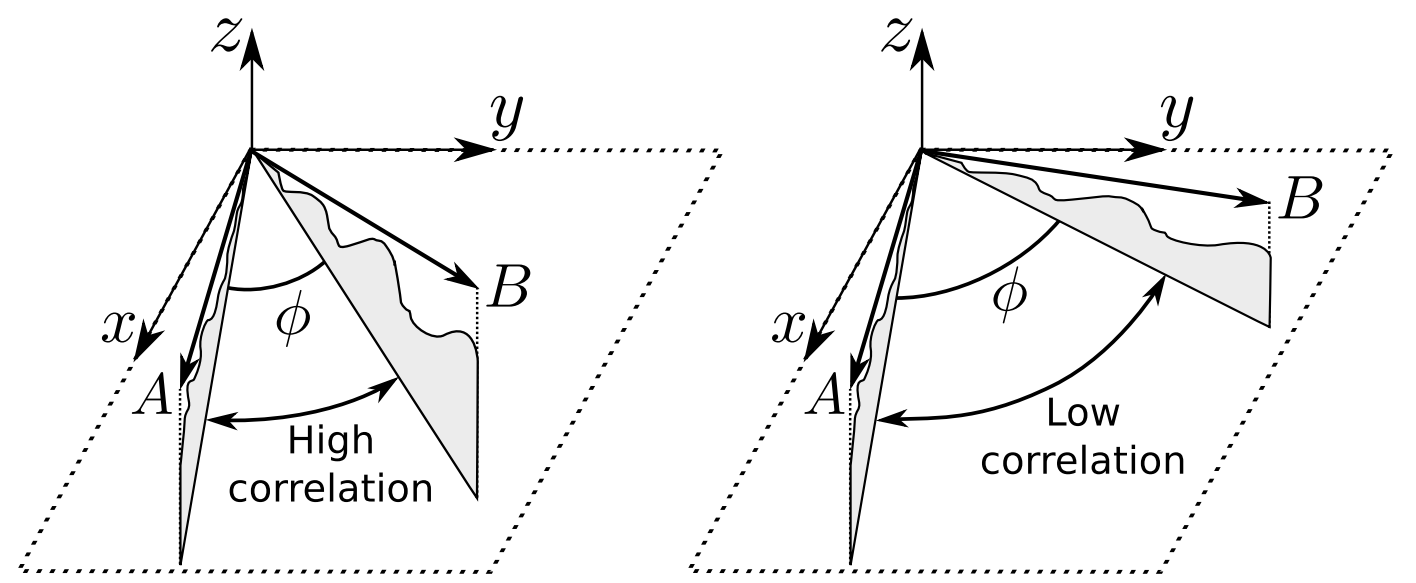

Figure 2. Illustration of the azimuthal correlation between two directions $A$ and $B$ in the bistatic configuration.

The bistatic illumination function $S(F, A \cap B)$ gives the probability that the point $F\left(\zeta_{0}, \gamma_{0 x}, \gamma_{0 y}\right)$ is visible simultaneously from both $\mathrm{A}$ and $\mathrm{B}$.

\begin{tabular}{|c|c|}
\hline Probability & Associated event \\
\hline$S(F, A \cap B)$ & The point $F$ is visible from both directions $A$ and $B$ \\
\hline$S_{\gamma}\left(\gamma_{0 x}, \gamma_{0 y}, A \cap B\right)$ & The point $F$ is not self-shadowed for directions $A$ and $B$ \\
\hline$S_{\zeta}\left(\zeta_{0}, A \cap B\right)$ & $\begin{array}{l}\left.\text { The ray " } F A \text { " is above the surface at } \tau_{A} \in\right] 0, \infty[\text { and } \\
\left.\text { the ray " } F B \text { " is above the surface at } \tau_{B} \in\right] 0, \infty[\end{array}$ \\
\hline$S_{\zeta}\left(\zeta_{0}, B \mid A\right)$ & $\begin{array}{l}\left.\text { The ray " } F B \text { " is above the surface at } \tau_{B} \in\right] 0, \infty[ \\
\left.\text { given that the ray " } F A \text { " is above the surface at } \tau_{A} \in\right] 0, \infty[\end{array}$ \\
\hline$S\left(\zeta_{0}, \bar{B}_{\tau_{B}} \mid\left(B_{\tau_{B}-d \tau} \cap A\right)\right)$ & $\begin{array}{l}\text { The ray " } F B \text { " intersects the surface at } \tau_{B} \\
\text { given that it is above the surface at } \tau_{B}-d \tau \text { and } \\
\left.\text { that the ray " } F A \text { " is above the surface at } \tau_{A} \in\right] 0, \infty[\end{array}$ \\
\hline
\end{tabular}

Bourlier et al. $[5,6]$ distinguished two cases.

The first case $\phi=0$. This case is equivalent to an in-plane configuration. In this case, the events $A$ and $B$ are correlated because the fact that the two rays are above the surface is determined by the fact that only the lowest ray is above the surface. Thus, only the lowest direction with the smallest probability needs to be considered

$$
S(F, A \cap B)=\min (S(F, A), S(F, B))
$$

The second case $\phi \in] 0, \pi]$. The events associated to $A$ and $B$ are then assumed to be independent (the probabilities can be separately estimated and multiplied 
between them), leading to

$$
S(F, A \cap B)=S(F, A) S(F, B)
$$

However, if $A$ and $B$ are close (Figure 2), the events can be strongly correlated and the above equation does not hold. This occurs when the absolute azimuthal difference $\phi$ is small. The next subsection presents a way to take into account this correlation.

\subsection{Inclusion of the Azimuthal Correlation}

Since the surface heights $\zeta$ and the slopes $\gamma$ are independent random variables, we can write

$$
S(F, A \cap B)=S_{\zeta}\left(\zeta_{0}, A \cap B\right) S_{\gamma}\left(\gamma_{0 x}, \gamma_{0 y}, A \cap B\right)
$$

The computation of $S_{\gamma}\left(\gamma_{0 x}, \gamma_{0 y}, A \cap B\right)$, corresponding to the probability that a facet with slopes $\left(\gamma_{0 x}, \gamma_{0 y}\right)$ is not self-shadowed simultaneously from the points $A$ and $B$, is straightforward and is explained in Section 2.3.1.

The most difficult part concerns the derivation of $S_{\zeta}\left(\zeta_{0}, A \cap B\right)$, presented in subsection 2.3.2.

\subsubsection{Derivation of $S_{\gamma}\left(\gamma_{0 x}, \gamma_{0 y}, A \cap B\right)$}

The probability that a facet with slopes $\left(\gamma_{0 x}, \gamma_{0 y}\right)$ is not self-shadowed for directions $A$ and $B$ is given by

$$
S_{\gamma}\left(\gamma_{0 x}, \gamma_{0 y}, A \cap B\right)=\Upsilon\left(\mu_{A}-\gamma_{A}, \mu_{B}-\gamma_{B}\right)
$$

where $\gamma_{A}$ and $\gamma_{B}$ are the slopes projected in the directions $A$ and $B$, respectively, as

$$
\begin{aligned}
& \gamma_{A}=\cos \phi_{A} \gamma_{0 x}+\sin \phi_{A} \gamma_{0 y} \\
& \gamma_{B}=\cos \phi_{B} \gamma_{0 x}+\sin \phi_{B} \gamma_{0 y}
\end{aligned}
$$

and $\Upsilon(x, y)$ is the two-dimensional Heaviside function

$$
\Upsilon(x, y)= \begin{cases}1 & \text { if } x, y>0 \\ 0 & \text { otherwise }\end{cases}
$$

It is important to note that since the Heaviside function has binary values, it can be written as a separable product of two one-variable Heaviside functions, leading to

$$
\begin{aligned}
S_{\gamma}\left(\gamma_{0 x}, \gamma_{0 y}, A \cap B\right) & =\Upsilon\left(\mu_{A}-\gamma_{A}\right) \Upsilon\left(\mu_{B}-\gamma_{B}\right) \\
& =S_{\gamma}\left(\gamma_{0 x}, \gamma_{0 y}, A\right) S_{\gamma}\left(\gamma_{0 x}, \gamma_{0 y}, B\right)
\end{aligned}
$$

The calculation of the average illumination function over the slopes $\left(\gamma_{0 x}, \gamma_{0 y}\right)$ 
requires to compute $\bar{S}_{\gamma}(A \cap B)$, which is defined as

$$
\begin{aligned}
& \bar{S}_{\gamma}(A \cap B) \\
= & \int_{-\infty}^{+\infty} \int_{-\infty}^{+\infty} \Upsilon\left(\mu_{A}-\gamma_{A}\right) \Upsilon\left(\mu_{B}-\gamma_{B}\right) p_{\gamma}\left(\gamma_{0 x}, \gamma_{0 y}\right) d \gamma_{0 x} d \gamma_{0 y}
\end{aligned}
$$

If the surface is isotropic $\left(\sigma_{\gamma_{0 x}}=\sigma_{\gamma_{0 y}} \equiv \sigma_{\gamma}\right)$, the slope distribution is separable. For Gaussian statistics, it is then written as

$$
\begin{aligned}
p_{\gamma}\left(\gamma_{0 x}, \gamma_{0 y}\right) & =\frac{1}{2 \pi \sigma_{\gamma}^{2}} \exp \left(-\frac{\gamma_{0 x}^{2}+\gamma_{0 y}^{2}}{2 \sigma_{\gamma}^{2}}\right) \\
& =\frac{1}{\sqrt{2 \pi} \sigma_{\gamma}} \exp \left(-\frac{\gamma_{0 x}^{2}}{2 \sigma_{\gamma}^{2}}\right) \frac{1}{\sqrt{2 \pi} \sigma_{\gamma}} \exp \left(-\frac{\gamma_{0 y}^{2}}{2 \sigma_{\gamma}^{2}}\right) \\
& =p_{\gamma}\left(\gamma_{0 x}\right) p_{\gamma}\left(\gamma_{0 x}\right)
\end{aligned}
$$

and the surface parametrization can be rotated so that $\gamma_{A}=\gamma_{0 x}$ and $\gamma_{B}=\cos (\phi) \gamma_{0 x}+\sin (\phi) \gamma_{0 y}$. In [6], an integration is done analytically and the last one numerically:

$$
\begin{aligned}
& \bar{S}_{\gamma}(A \cap B) \\
= & \int_{-\infty}^{+\infty} \int_{-\infty}^{+\infty} \Upsilon\left(\mu_{A}-\gamma_{0 x}\right) \Upsilon\left(\mu_{B}-\cos \phi \gamma_{0 x}-\sin \phi \gamma_{0 y}\right) p_{\gamma}\left(\gamma_{0 x}\right) p_{\gamma}\left(\gamma_{0 y}\right) d \gamma_{0 x} d \gamma_{0 y} \\
= & \int_{-\infty}^{+\infty} \Upsilon\left(\mu_{A}-\gamma_{0 x}\right) p_{\gamma}\left(\gamma_{0 x}\right) \int_{-\infty}^{+\infty} \Upsilon\left(\mu_{B}-\cos \phi \gamma_{0 x}-\sin \phi \gamma_{0 y}\right) p_{\gamma}\left(\gamma_{0 y}\right) d \gamma_{0 y} d \gamma_{0 x} \\
= & \int_{-\infty}^{+\infty} \Upsilon\left(\mu_{A}-\gamma_{0 x}\right) p_{\gamma}\left(\gamma_{0 x}\right) P_{\gamma}\left(\frac{\mu_{B}-\cos \phi \gamma_{0 x}}{\sin \phi}\right) d \gamma_{0 x}
\end{aligned}
$$

where $P_{\gamma}(x)=[1-\operatorname{erfc}(x)] / 2$.

\subsubsection{Derivation of $S_{\zeta}\left(\zeta_{0}, A \cap B\right)$}

The probability that the rays coming from $F$ and propagating towards $A$ and $B$ do not intersect the surface can be expressed from the Bayes conditional probability theorem as

$$
S_{\zeta}\left(\zeta_{0}, A \cap B\right)=S_{\zeta}\left(\zeta_{0}, A\right) S_{\zeta}\left(\zeta_{0}, B \mid A\right)
$$

where $S_{\zeta}\left(\zeta_{0}, A\right)$ is the monostatic illumination function expressed from Eq. (2). The conditional probability $S_{\zeta}\left(\zeta_{0}, B \mid A\right)$ is the probability that the ray " $F B$ " does not intersect the surface given that the ray " $F A$ " does not as well. The purpose of this subsection is to find an approximation of this probability.

The event $A$ : the ray " $F A$ " is not blocked by the surface provides the following information: "All points of the surface below $A$ have heights which are bounded by the height of A at the corresponding abscissa". We introduce this event in Eq. (2)

$$
S_{\zeta}\left(\zeta_{0}, B \mid A\right)=\exp \left[-\int_{0}^{\infty} S_{\zeta}\left(\zeta_{0}, \bar{B}_{\tau_{B}} \mid\left(B_{\tau_{B}-d \tau} \cap A\right)\right) d \tau_{B}\right]
$$


in which the function $S_{\zeta}\left(\zeta_{0}, \bar{B}_{\tau_{B}} \mid B_{\tau_{B}-d \tau}\right)$ is replaced by $S_{\zeta}\left(\zeta_{0}, \bar{B}_{\tau_{B}} \mid\left(B_{\tau_{B}-d \tau} \cap A\right)\right)$ by using the information provided by the event A.

Modelling the information provided by the event $A$ is not trivial for two reasons. First, this information is not punctual (only one point of the surface) but onedimensional (set of points along the cross section of the surface) and thus, it is complicated to model and to apply. Second, this information is related to the surface behaviour and it has to be transformed into information useful for the computation of the intersection with the ray " $F B$ ". In the following, we propose a heuristic way to model this information and we show how to use it to model the probability $S_{\zeta}\left(\zeta_{0}, \bar{B}_{\tau_{B}} \mid\left(B_{\tau_{B}-d \tau} \cap A\right)\right)$.

\subsubsection{Derivation of the Conditional Probability $S_{\zeta}\left(\zeta_{0}, \bar{B}_{\tau_{B}} \mid\left(B_{\tau_{B}-d \tau} \cap A\right)\right)$}

For each point of the ray " $F B$ ", instead of using the entire information available along the ray " $F A$ ", we only use the information provided by the point of " $F A$ " in the direction orthogonal to the ray " $F B$ " along the surface, as shown in Figure 3.

We note $\tau_{A}$ the abscissa of only one point of " $F A$ " and we approximate $S_{\zeta}\left(\zeta_{0}, \bar{B}_{\tau_{B}} \mid\left(B_{\tau_{B}-d \tau} \cap A\right)\right) \approx S_{\zeta}\left(\zeta_{0}, \bar{B}_{\tau_{B}} \mid\left(B_{\tau_{B}-d \tau} \cap A_{\tau_{A}}\right)\right)$. An important consequence of this approximation is that we need to choose $A$ and $B$ such that $\mu_{A} \leq \mu_{B}$. Indeed, in an in-plane configuration, if $\mu_{A}>\mu_{B}$ then $B_{\tau_{B}-d \tau} \Rightarrow A_{\tau_{A}}$ and thus $S_{\zeta}\left(\zeta_{0}, \bar{B}_{\tau_{B}} \mid\left(B_{\tau_{B}-d \tau} \cap A_{\tau_{A}}\right)\right)=S_{\zeta}\left(\zeta_{0}, \bar{B}_{\tau_{B}} \mid B_{\tau_{B}-d \tau}\right)$ and the information provided by the ray " $F A$ ' is not used. From now on, we assume that $\mu_{A} \leq \mu_{B}$.

Note that this approximation is very similar to that used by Smith [2] for the derivation of the monostatic 1-D illumination function. Indeed, for the monostatic case, the probability $S_{\zeta}\left(\zeta_{0}, \bar{B}_{\tau_{B}} \mid B_{\tau_{B}-d \tau}\right)$ from Equation (3) expresses the probability that the ray intersects the surface at the distance $\tau_{B}$ given that the ray is above the surface at the distance $\tau_{B}-d \tau$. Considering that the surface is below the ray at the abscissa $\tau_{B}-d \tau$ is an approximation of the event that the surface must be below the ray in the interval $\left[0 ; \tau_{B}[\right.$. Since modelling information over the interval $\left[0 ; \tau_{B}[\right.$ is complicated, Smith replaced this information only by the single point $\tau_{B}-d \tau$. He replaced the event $B_{\left[0, \tau_{B}[\right.}$ by $B_{\tau_{B}-d \tau}$. Then, our approximation is in the same spirit but is applied to the other ray. We chose a point below " $F A$ " in the direction orthogonal to " $F B$ " so that we have $A_{\tau_{A}}\left|B_{\tau_{B}-d \tau} \approx A_{\tau_{A}}\right| B_{\tau_{B}}$, which will allow us to simplify Equation (16).

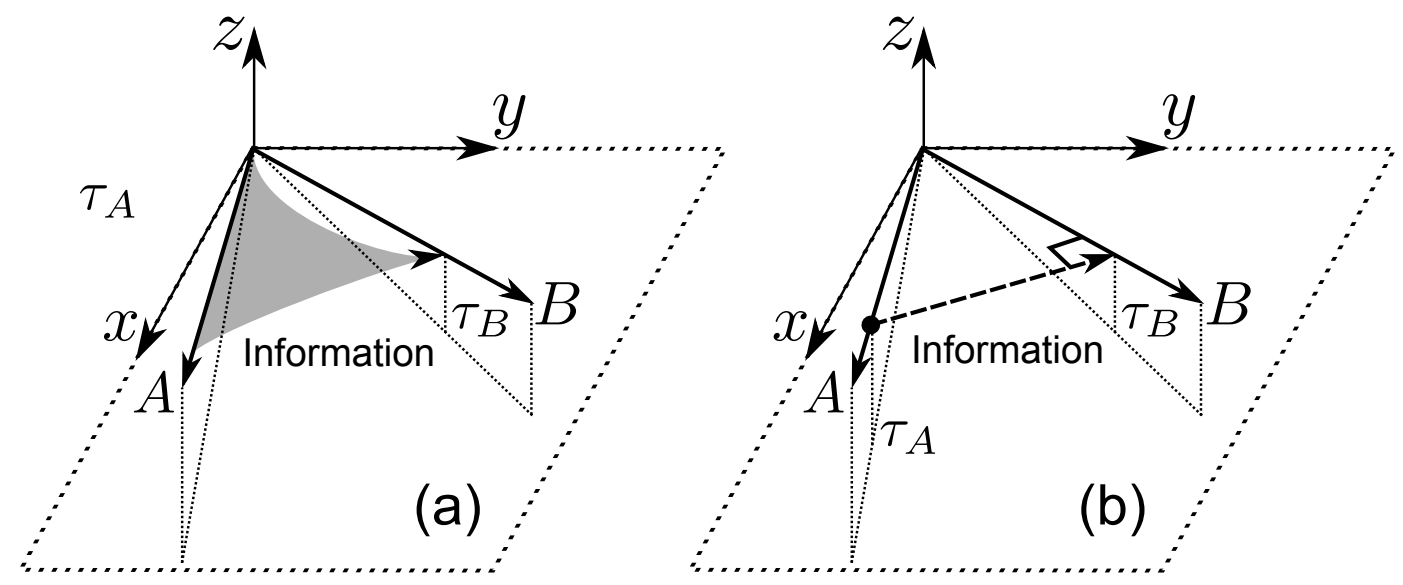

Figure 3. (a) The non-shadowing for direction $A$ provides information for all points of the surface below the ray. (b) In the computation of $B$, we approximate this information by considering only one point below $A$, which corresponds to the orthogonal projection onto the ray " $F B$ " at the point of abscissa $\tau_{B}$.

If $\phi \in\left[\frac{\pi}{2}, \pi\right]$, then for all abscissae $\tau_{B} \geq 0$, the point below " $F A$ " is located at 
an infinite distance and does not provide any information. In this case, we neglect the information provided by event $A$ and we get $S_{\zeta}\left(\zeta_{0}, \bar{B}_{\tau_{B}} \mid\left(B_{\tau_{B}-d \tau} \cap A\right)\right)=$ $S_{\zeta}\left(\zeta_{0}, \bar{B}_{\tau_{B}} \mid B_{\tau_{B}-d \tau}\right)$ and $S_{\zeta}\left(\zeta_{0}, B \mid A\right)=S_{\zeta}\left(\zeta_{0}, B\right)$. In the following, we consider the case where $\phi \in\left[0, \frac{\pi}{2}[\right.$.

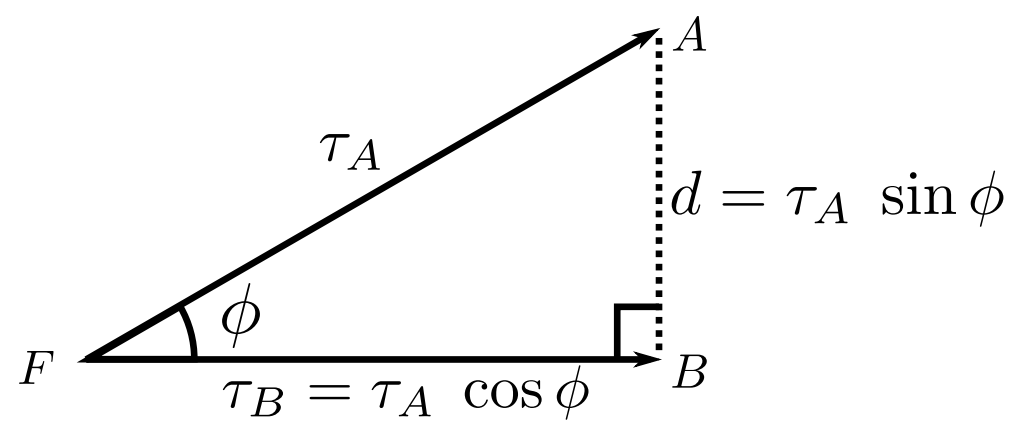

Figure 4. Distance between the points of abscissa $\tau_{A}$ and $\tau_{B}$ on rays $A$ and $B$, respectively.

If $\phi \in\left[0, \frac{\pi}{2}\right.$ [, the point below " $F A$ " corresponding to abscissa $\tau_{B}$ on " $F B$ " has an abscissa $\tau_{A}=\tau_{B} / \cos \phi$ on $A$, and the distance between the points is $d=\tau_{B} \tan \phi$ (see Fig. 4). In addition, since the point is below the ray " $F A$ ", its height is upperbounded by $\zeta_{\tau_{A}}<\zeta_{0}+\tau_{A} \mu_{A}=\zeta_{0}+\tau_{B} \mu_{A} / \cos \phi$. Let us denote $A_{\tau_{A}}$ this event. By using Bayes's conditional probability theorem, we expand

$$
\begin{aligned}
S_{\zeta}\left(\zeta_{0}, \bar{B}_{\tau_{B}} \mid\left(B_{\tau_{B}-d \tau} \cap A_{\tau_{A}}\right)\right) & =S_{\zeta}\left(\zeta_{0},\left(\bar{B}_{\tau_{B}} \mid A_{\tau_{A}}\right) \mid B_{\tau_{B}-d \tau}\right) \\
& =S_{\zeta}\left(\zeta_{0}, \bar{B}_{\tau_{B}} \mid B_{\tau_{B}-d \tau}\right) \frac{S_{\zeta}\left(\zeta_{0},\left(A_{\tau_{A}} \mid \bar{B}_{\tau_{B}}\right) \mid B_{\tau_{B}-d \tau}\right)}{S_{\zeta}\left(\zeta_{0}, A_{\tau_{A}} \mid B_{\tau_{B}-d \tau}\right)} \\
& =S_{\zeta}\left(\zeta_{0}, \bar{B}_{\tau_{B}} \mid B_{\tau_{B}-d \tau}\right) \frac{S_{\zeta}\left(\zeta_{0}, A_{\tau_{A}} \mid\left(\bar{B}_{\tau_{B}} \cap B_{\tau_{B}-d \tau}\right)\right)}{S_{\zeta}\left(\zeta_{0}, A_{\tau_{A}} \mid B_{\tau_{B}-d \tau}\right)}
\end{aligned}
$$

To simplify this equation, we use the fact that $\bar{B}_{\tau_{B}} \cap B_{\tau_{B}-d \tau}=\bar{B}_{\tau_{B}}$. Furthermore, since we have chosen a point below " $F A$ " which is located in the direction orthogonal to " $F B$ ", we can make the approximation $A_{\tau_{A}}\left|B_{\tau_{B}-d \tau} \approx A_{\tau_{A}}\right| B_{\tau_{B}}$. We get

$$
S_{\zeta}\left(\zeta_{0}, \bar{B}_{\tau_{B}} \mid\left(B_{\tau_{B}-d \tau} \cap A_{\tau_{A}}\right)\right)=S_{\zeta}\left(\zeta_{0}, \bar{B}_{\tau_{B}} \mid B_{\tau_{B}-d \tau}\right) \frac{S_{\zeta}\left(\zeta_{0}, A_{\tau_{A}} \mid \bar{B}_{\tau_{B}}\right)}{S_{\zeta}\left(\zeta_{0}, A_{\tau_{A}} \mid B_{\tau_{B}}\right)}
$$

where $S_{\zeta}\left(\zeta_{0}, \bar{B}_{\tau_{B}} \mid B_{\tau_{B}-d \tau}\right)$ is the Smith monostatic illumination function from Equation (3), which does not account for events associated to the ray " $F A$ ".

$S_{\zeta}\left(\zeta_{0}, A_{\tau_{A}} \mid \bar{B}_{\tau_{B}}\right)$ is the probability that the surface is below the ray " $F A$ " at $\tau_{A}$ - the height of the surface is in interval ] $-\infty, \zeta_{\tau_{A}}$ [ below $A_{\tau_{A}}$ - given that the ray " $F B$ " crosses the surface at $\tau_{B}$ - the height of the surface is $\zeta_{\tau_{B}}=\zeta_{0}+\tau_{B} \mu_{B}$ below $B_{\tau_{B}}$. The information provided by the point $B_{\tau_{B}}$ provides information about the height distribution below $A_{\tau_{A}}$. The height $\mathrm{PDF} p_{\zeta}$ at this point is transformed into a conditional PDF $p_{\zeta \mid \zeta_{\tau_{B}}}$. Assuming that $p_{\zeta \mid \zeta_{\tau_{B}}}$ is known for a specific height $\zeta_{\tau_{B}}$, then

$$
\begin{aligned}
S_{\zeta}\left(\zeta_{0}, A_{\tau_{A}} \mid \bar{B}_{\tau_{B}}\right) & =\int_{-\infty}^{\zeta_{\tau_{A}}} p_{\zeta \mid \zeta_{\tau_{B}}}(\zeta) d \zeta \\
& =P_{\zeta \mid \zeta_{\tau_{B}}}\left(\zeta_{\tau_{A}}\right)
\end{aligned}
$$


$S_{\zeta}\left(\zeta_{0}, A_{\tau_{A}} \mid B_{\tau_{B}}\right)$ is the probability that the surface is below the ray " $F A$ " at $\tau_{A}$ given that the surface is below the ray " $F B$ " at $\tau_{B}$. The event $B_{\tau_{B}}$ fixes the height $\zeta_{\tau_{B}} \in\left[-\infty, \zeta_{0}+\tau_{B} \mu_{B}\left[\right.\right.$ weighted by the height PDF $p_{\zeta}$, and then

$$
\begin{aligned}
S_{\zeta}\left(\zeta_{0}, A_{\tau_{A}} \mid B_{\tau_{B}}\right) & =\int_{-\infty}^{\zeta_{0}+\tau_{B} \mu_{B}} \frac{p_{\zeta}\left(\zeta_{\tau_{B}}\right)}{P_{\zeta}\left(\zeta_{0}+\tau_{B} \mu_{B}\right)} S_{\zeta}\left(\zeta_{0}, A_{\tau_{A}} \mid \bar{B}_{\tau_{B}}\right) d \zeta_{\tau_{B}} \\
& =\int_{-\infty}^{\zeta_{0}+\tau_{B} \mu_{B}} \frac{p_{\zeta}\left(\zeta_{\tau_{B}}\right)}{P_{\zeta}\left(\zeta_{0}+\tau_{B} \mu_{B}\right)} P_{\zeta \mid \zeta_{\tau_{B}}}\left(\zeta_{\tau_{A}}\right) d \zeta_{\tau_{B}}
\end{aligned}
$$

\subsubsection{Derivation of $p_{\zeta \mid \zeta_{\tau_{B}}}$}

Equations (17) and (18) require the knowledge of the conditional PDF $p_{\zeta \mid \zeta_{\tau_{B}}}$. From the height $\zeta_{\tau_{B}}$ and the slope $\gamma$, the height at a distance $d$ can be approximated from a first-order Taylor series expansion as $\zeta_{\tau_{B}}+\gamma d$. Since the PDF slope follows $\mathcal{N}\left(0, \sigma_{\gamma}^{2}\right)$, the PDF of $\zeta_{\tau_{B}}+\gamma d$ follows $\mathcal{N}\left(\zeta_{\tau_{B}},\left(d \sigma_{\gamma}\right)^{2}\right)$. This is illustrated in Figure 5. Thus, a point located along $B$ at a distance $d$ at the height $\zeta_{\tau_{B}}$ can be interpreted as a measure with a Gaussian uncertainty.

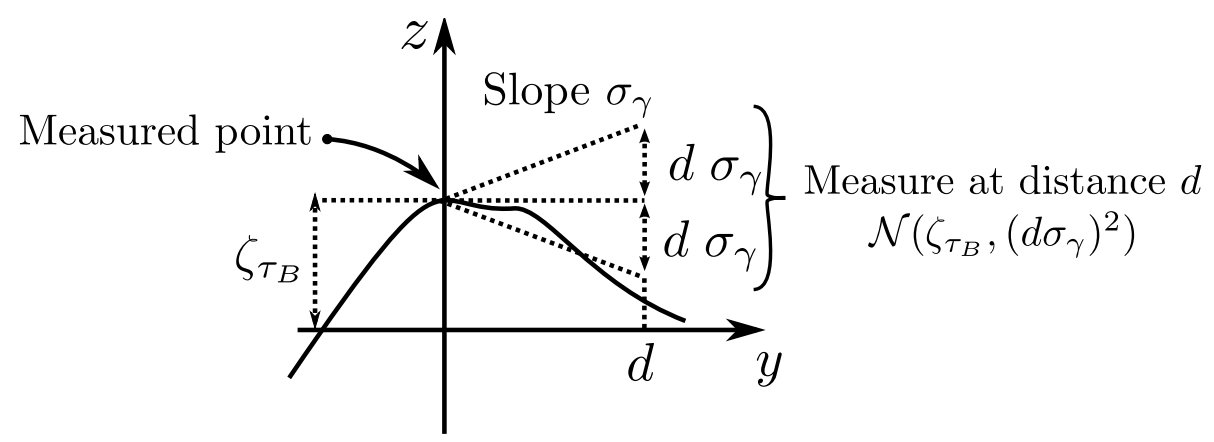

(a)

Figure 5. The measure of a point of the surface provides information on its neighbourhood.

If two independent measures $x_{1} \sim \mathcal{N}\left(m_{1}, \sigma_{1}^{2}\right)$ and $x_{2} \sim \mathcal{N}\left(m_{2}, \sigma_{2}^{2}\right)$ are fused with the minimum-variance unbiased estimator method [7], then the result is $x \sim \mathcal{N}\left(m, \sigma^{2}\right)$ with a mean value $m=\left(m_{1} / \sigma_{1}^{2}+m_{2} / \sigma_{2}^{2}\right) /\left(1 / \sigma_{1}^{2}+1 / \sigma_{2}^{2}\right)$ and a variance $\sigma^{2}=1 /\left(1 / \sigma_{1}^{2}+1 / \sigma_{2}^{2}\right)$.

Then, assuming that $\zeta \sim \mathcal{N}\left(0, \sigma_{\zeta}^{2}\right)$ and $\zeta_{\tau_{B}}+\gamma d \sim \mathcal{N}\left(\zeta_{\tau_{B}},\left(d \sigma_{\gamma}\right)^{2}\right)$ are independent measures, the conditional PDF $p_{\zeta \mid \zeta_{\tau_{B}}}\left(\zeta_{\tau_{A}}\right)$ follows $\mathcal{N}\left(m_{\zeta_{\tau_{A}} \mid \zeta_{\tau_{B}}}, \sigma_{\zeta_{\tau_{A}} \mid \zeta_{\tau_{B}}}^{2}\right)$ with mean

$$
m_{\zeta_{\tau_{A}} \mid \zeta_{\tau_{B}}}=\frac{\zeta_{\tau_{B}}}{1+\left(\frac{d \sigma_{\gamma}}{\sigma_{\zeta}}\right)^{2}}
$$

and variance

$$
\sigma_{\zeta_{\tau_{A}} \mid \zeta_{\tau_{B}}}^{2}=\frac{\left(\sigma_{\zeta} d \sigma_{\gamma}\right)^{2}}{\sigma_{\zeta}^{2}+\left(d \sigma_{\gamma}\right)^{2}}
$$

The associated cumulative distribution function used in Equations (17) and (18) is

$$
P_{\zeta \mid \zeta_{\tau_{B}}}\left(\zeta_{\tau_{A}}\right)=1-\frac{1}{2} \operatorname{erfc}\left(\frac{\zeta_{\tau_{A}}-m_{\zeta_{\tau_{A}} \mid \zeta_{\tau_{B}}}}{\sqrt{2} \sigma_{\zeta_{\tau_{A}} \mid \zeta_{\tau_{B}}}}\right)
$$




\subsubsection{Properties of the Azimuthal Correlation in $S_{\zeta}\left(\zeta_{0}, A \cap B\right)$}

Our modelling of the azimuthal correlation in the term $S_{\zeta}\left(\zeta_{0}, A \cap B\right)$ is consistent with the in-plane and no correlation configurations for which the results are known.

- Case with no correlation $\phi \in\left[\frac{\pi}{2}, \pi\right]$. In subsection 2.3 .3 , we explain why the information provided by $A$ should be neglected according to Smith's model if $\phi \in\left[\frac{\pi}{2}, \pi\right]$. In this case, our model with azimuthal correlation comes back to the separable model with no correlation $S_{\zeta}\left(\zeta_{0}, A \cap B\right)=S_{\zeta}\left(\zeta_{0}, A\right) S_{\zeta}\left(\zeta_{0}, B\right)$.

- Continuity toward the case with no correlation $\phi \rightarrow \frac{\pi}{2}$. In Equation (18), the distance between the points $A_{\tau_{A}}$ and $B_{\tau_{B}}$ is defined by $d=\tau_{B} \tan \phi$ and $\lim _{\phi \rightarrow \frac{\pi}{2}} d=\infty$. Thus, the variance of the second measure from subsection 2.3.4 tends toward infinity $\lim _{\phi \rightarrow \frac{\pi}{2}}\left(\sigma_{\gamma} d\right)^{2}=\infty$, and the estimation $\lim _{\phi \rightarrow \frac{\pi}{2}} \mathcal{N}\left(m_{\zeta_{\tau_{A}} \mid \zeta_{\tau_{B}}}, \sigma_{\zeta_{\tau_{A}} \mid \zeta_{\tau_{B}}}^{2}\right)=\mathcal{N}\left(0, \sigma_{\zeta}^{2}\right)$ tends toward the surface distribution. In this case, no information is provided by the other ray, and $\lim _{\phi \rightarrow \frac{\pi}{2}} S_{\zeta}\left(\zeta_{0}, A_{\tau_{A}} \mid \bar{B}_{\tau_{B}}\right)=\lim _{\phi \rightarrow \frac{\pi}{2}} S_{\zeta}\left(\zeta_{0}, A_{\tau_{A}} \mid B_{\tau_{B}}\right)=$ $P_{\zeta}\left(\zeta_{\tau_{A}}\right)$ and $\lim _{\phi \rightarrow \frac{\pi}{2}} S_{\zeta}\left(\zeta_{0}, \bar{B}_{\tau_{B}} \mid\left(B_{\tau_{B}-d \tau} \cap A_{\tau_{A}}\right)\right)=S_{\zeta}\left(\zeta_{0}, \bar{B}_{\tau_{B}} \mid B_{\tau_{B}-d \tau}\right)$. Thus, $\lim _{\phi \rightarrow \frac{\pi}{2}} S_{\zeta}\left(\zeta_{0}, B \mid A\right)=S_{\zeta}\left(\zeta_{0}, B\right)$, and the model tends toward the separable model with no correlation as the azimuthal angle approaches $\frac{\pi}{2}$.

- Case with an in-plane configuration $\phi=0$. In this case, the points below $A$ coincide with the points below $B$. They share the same abscissae parametrization $\tau=\tau_{A}=\tau_{B}$, the distance to the nearest neighbour is $d=0$ and the PDF $p_{\zeta_{\tau_{A}} \mid \zeta_{\tau_{B}}}$ is the Dirac delta distribution $\delta_{\zeta_{\tau_{B}}}$. The probability $S_{\zeta}\left(\zeta_{0}, A_{\tau_{A}} \mid \bar{B}_{\tau_{B}}\right)$ from Equation (16) becomes

$$
\begin{aligned}
S_{\zeta}\left(\zeta_{0}, A_{\tau_{A}} \mid \bar{B}_{\tau_{B}}\right) & =P_{\zeta_{\tau_{A}} \mid \zeta_{\tau_{B}}}\left(\zeta_{\tau_{A}}\right) \\
& =\int_{-\infty}^{\zeta_{\tau_{A}}} \delta_{\zeta_{\tau_{B}}}(\zeta) d \zeta \\
& =0
\end{aligned}
$$

since $A$ and $B$ are chosen such that $\mu_{A} \leq \mu_{B}$, i.e., the value $\zeta_{\tau_{B}}$ is above $\zeta_{\tau_{A}}$ and the Dirac delta evaluates to 0 . Because $S_{\zeta}\left(\zeta_{0}, A_{\tau_{A}} \mid B_{\tau_{B}}\right)$ is the average of $P_{\zeta_{\tau_{A}} \mid \zeta_{\tau_{B}}}\left(\zeta_{\tau_{A}}\right)$ in $\left.]-\infty, \zeta_{\tau_{B}}\right]$, it is strictly positive. Thus, Equation (16) equals 0. The conditional probability is then $S_{\zeta}\left(\zeta_{0}, B \mid A\right)=\exp (0)=1$, and the probability of the intersection is $S_{\zeta}\left(\zeta_{0}, A \cap B\right)=S_{\zeta}\left(\zeta_{0}, A\right)$. This is the expected result of the bistatic in-plane configuration where only the lowest direction is considered.

\subsubsection{Development}

By using the result of Equation (16) in Equation (15), we get

$$
S_{\zeta}\left(\zeta_{0}, B \mid A\right)=\exp \left[-\int_{0}^{\infty} S_{\zeta}\left(\zeta_{0}, \bar{B}_{\tau_{B}} \mid B_{\tau_{B}-d \tau}\right) \frac{S_{\zeta}\left(\zeta_{0}, A_{\tau_{A}} \mid \bar{B}_{\tau_{B}}\right)}{S_{\zeta}\left(\zeta_{0}, A_{\tau_{A}} \mid B_{\tau_{B}}\right)} d \tau_{B}\right]
$$

To simplify the expression, we introduce the correction coefficient

$$
r\left(\tau_{B}\right)=\frac{S_{\zeta}\left(\zeta_{0}, A_{\tau_{A}} \mid \bar{B}_{\tau_{B}}\right)}{S_{\zeta}\left(\zeta_{0}, A_{\tau_{A}} \mid B_{\tau_{B}}\right)}
$$


and we write the probability

$$
\begin{aligned}
S_{\zeta}\left(\zeta_{0}, B \mid A\right) & =\exp \left[-\int_{0}^{\infty} S_{\zeta}\left(\zeta_{0}, \bar{B}_{\tau_{B}} \mid B_{\tau_{B}-d \tau}\right) r\left(\tau_{B}\right) d \tau_{B}\right] \\
& =\exp \left[-\Lambda\left(\mu_{B}\right) \mu_{B} \int_{0}^{\infty} \frac{p_{\zeta}\left(\zeta_{0}+\tau_{B} \mu_{B}\right)}{P_{\zeta}\left(\zeta_{0}+\tau_{B} \mu_{B}\right)} r\left(\tau_{B}\right) d \tau_{B}\right] \\
& =\exp \left[-\Lambda\left(\mu_{B}\right) \mu_{B} r_{0} \int_{0}^{\infty} \frac{p_{\zeta}\left(\zeta_{0}+\tau_{B} \mu_{B}\right)}{P_{\zeta}\left(\zeta_{0}+\tau_{B} \mu_{B}\right)} d \tau_{B}\right]
\end{aligned}
$$

where the average correction coefficient is defined by

$$
r_{0}=\int_{0}^{\infty} \frac{p_{\zeta}\left(\zeta_{0}+\tau_{B} \mu_{B}\right)}{P_{\zeta}\left(\zeta_{0}+\tau_{B} \mu_{B}\right)} r\left(\tau_{B}\right) d \tau_{B} / \int_{0}^{\infty} \frac{p_{\zeta}\left(\zeta_{0}+\tau_{B} \mu_{B}\right)}{P_{\zeta}\left(\zeta_{0}+\tau_{B} \mu_{B}\right)} d \tau_{B}
$$

The introduction of the average correction coefficient allows us to integrate Equation (24) similarly to Equation (5) as

$$
S_{\zeta}\left(\zeta_{0}, B \mid A\right)=P_{\zeta}\left(\zeta_{0}\right)^{r_{0} \Lambda\left(\mu_{B}\right)}
$$

\subsubsection{Approximation of $r_{0}$}

The average correction coefficient $r_{0}$ results from an integration which has no analytical solution. In this section, we propose an analytical approximation. By developing $r$ given in Equation (23) with the definition of the probabilities from Equations (17) and (18), we rewrite Equation (25) as

$$
r_{0}=\int_{0}^{\infty} \frac{p_{\zeta}\left(\zeta_{0}+\tau_{B} \mu_{B}\right) P_{\zeta \mid \zeta_{\tau_{B}}}\left(\zeta_{\tau_{A}}\right)}{\int_{-\infty}^{\zeta_{0}+\mu_{B} \tau_{B}} p_{\zeta}\left(\zeta_{B}^{\prime}\right) P_{\zeta \mid \zeta_{B}^{\prime}\left(\tau_{B}\right)}\left(\zeta_{\tau_{A}}\right) d \zeta_{B}^{\prime}} d \tau_{B} / \int_{0}^{\infty} \frac{p_{\zeta}\left(\zeta_{0}+\tau_{B} \mu_{B}\right)}{P_{\zeta}\left(\zeta_{0}+\tau_{B} \mu_{B}\right)} d \tau_{B}
$$

where $P_{\zeta \mid \zeta_{\tau_{A}}}$ is the CDF given from Equation (21). The average correction coefficient is a multivariate function $r_{0}\left(\phi, \zeta_{0}, \mu_{A}, \mu_{B}, \sigma_{\gamma}\right)$. Thanks to our model properties, we know that $r_{0}(\phi=0)=0$ and $r_{0}(\phi=\pi / 2)=1$.

By computing $r_{0}$ numerically with Equation (27) provided by our model, we observe empirically that the sensitiveness to variable $\zeta_{0}$ is low.

Thanks to the surface horizontal scaling property, we have $r_{0}\left(\phi, \zeta_{0}, \mu_{A}, \mu_{B}, \sigma_{\gamma}\right)=$ $r_{0}\left(\phi, \zeta_{0}, \nu_{A}, \nu_{B}, 1 / \sqrt{2}\right)$, where $\nu_{A}=\mu_{A} / \sqrt{2} \sigma_{\gamma}$ and $\nu_{B}=\mu_{B} / \sqrt{2} \sigma_{\gamma}$ are the normalized slope.

Furthermore, we also observe that the sensitiveness to the variables $\nu_{A}$ and $\nu_{B}$ mainly depends on the difference $\nu_{B}-\nu_{A}$.

Thus, by approximating $\zeta_{0}=0$ and replacing parameters $\nu_{A}$ and $\nu_{B}$ by $\nu_{B}-$ $\nu_{A}$, we get a function of only 2 parameters for which we propose the following approximation

$$
r_{0}\left(\phi, \nu_{B}-\nu_{A}\right)= \begin{cases}\log \left(1+\alpha \phi^{\beta}\right) / \log \left(1+\alpha(\pi / 2)^{\beta}\right) & \text { if } \phi \in\left[0, \frac{\pi}{2}[\right. \\ 1 & \text { if } \phi \in\left[\frac{\pi}{2}, \pi\right]\end{cases}
$$

with $\alpha=0.17 /\left(\frac{\mu_{B}-\mu_{A}}{\sigma_{\gamma}}\right)^{10.49}$ and $\beta=8.85$. The numerical values $\alpha$ and $\beta$ were found with a non-linear optimization algorithm which optimizes the bistatic height 


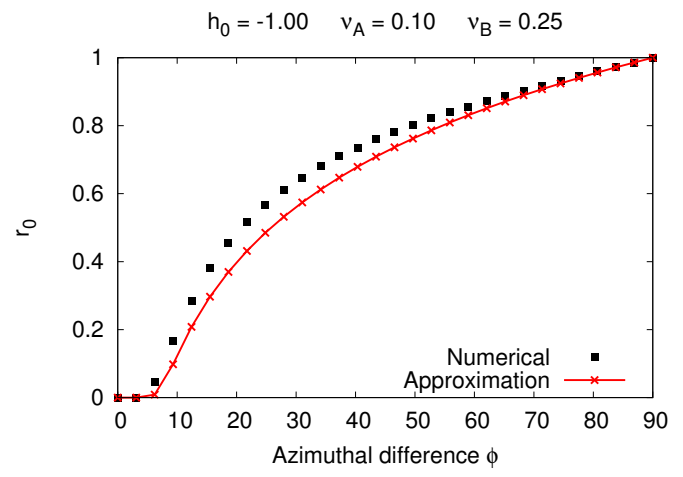

(a)

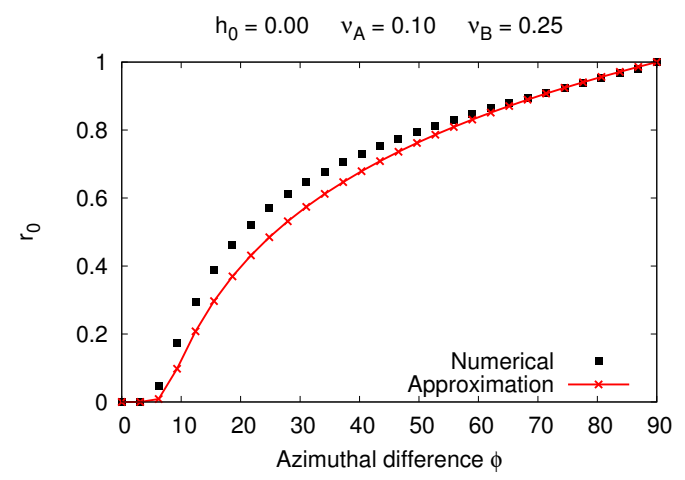

(c)

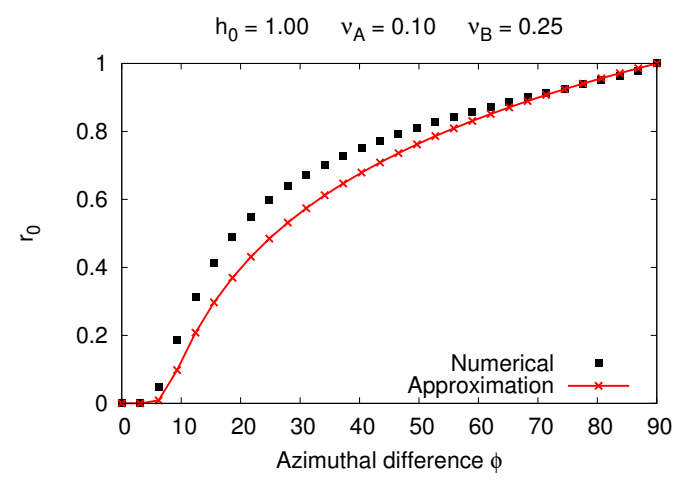

(e)

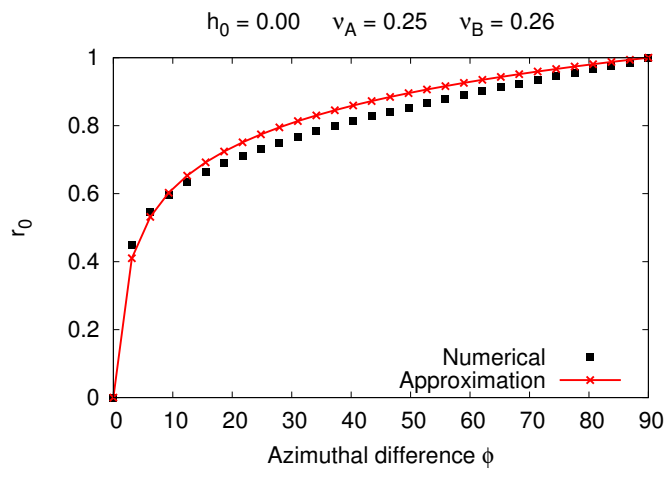

(b)

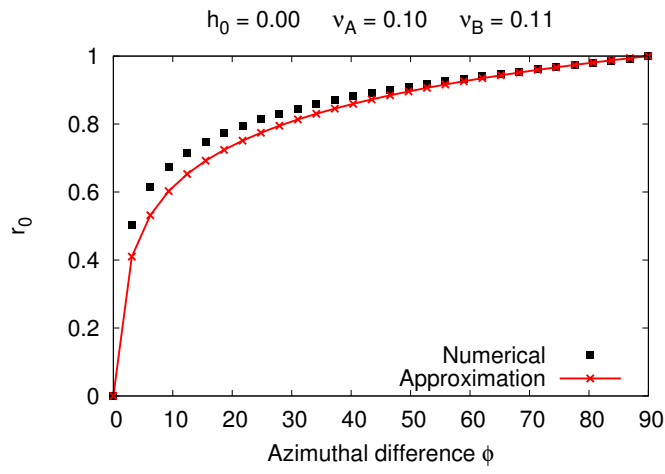

(d)

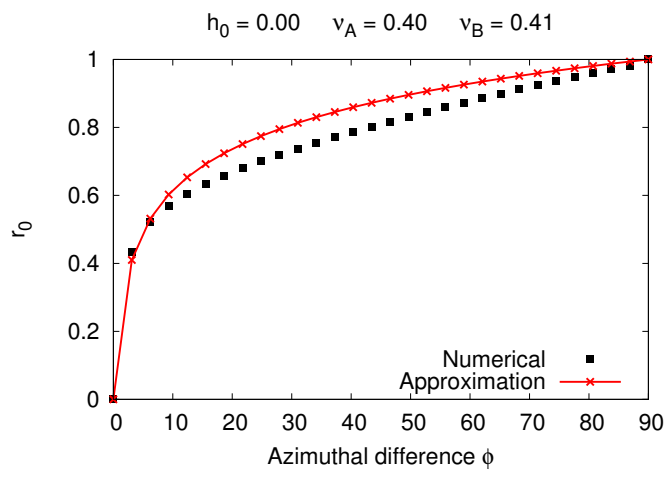

(f)

Figure 6. Average correction coefficient $r_{0}$ and our analytical approximation for different configurations. Variations of the normalized height $h_{0}=\zeta_{0} / \sqrt{2} \sigma_{\zeta}$ has a low impact on $r_{0}$ (a, c, e). It depends mostly on $\nu_{B}-\nu_{A}$, wich is equal to 0.15 and 0.01 in $(\mathrm{a}, \mathrm{c}, \mathrm{e})$ and in $(\mathrm{b}, \mathrm{d}, \mathrm{f})$, respectively.

illumination function $S_{\zeta}\left(\zeta_{0}, A\right) S_{\zeta}\left(\zeta_{0}, B \mid A\right)$. We compared the difference between the results obtained with our analytical approximation of $r_{0}$ with the results obtained with the numerically integrated $r_{0}$ from Equation (27). Our approximation produces a maximal error of $1.1 \%$ on the bistatic height illumination function. Comparisons between the numerically integrated $r_{0}$ and our approximation are shown in Figure 6. These results were computed with the code provided in the Appendix. Also, since $r_{0}$ does not depend on the surface heights, we can easily 


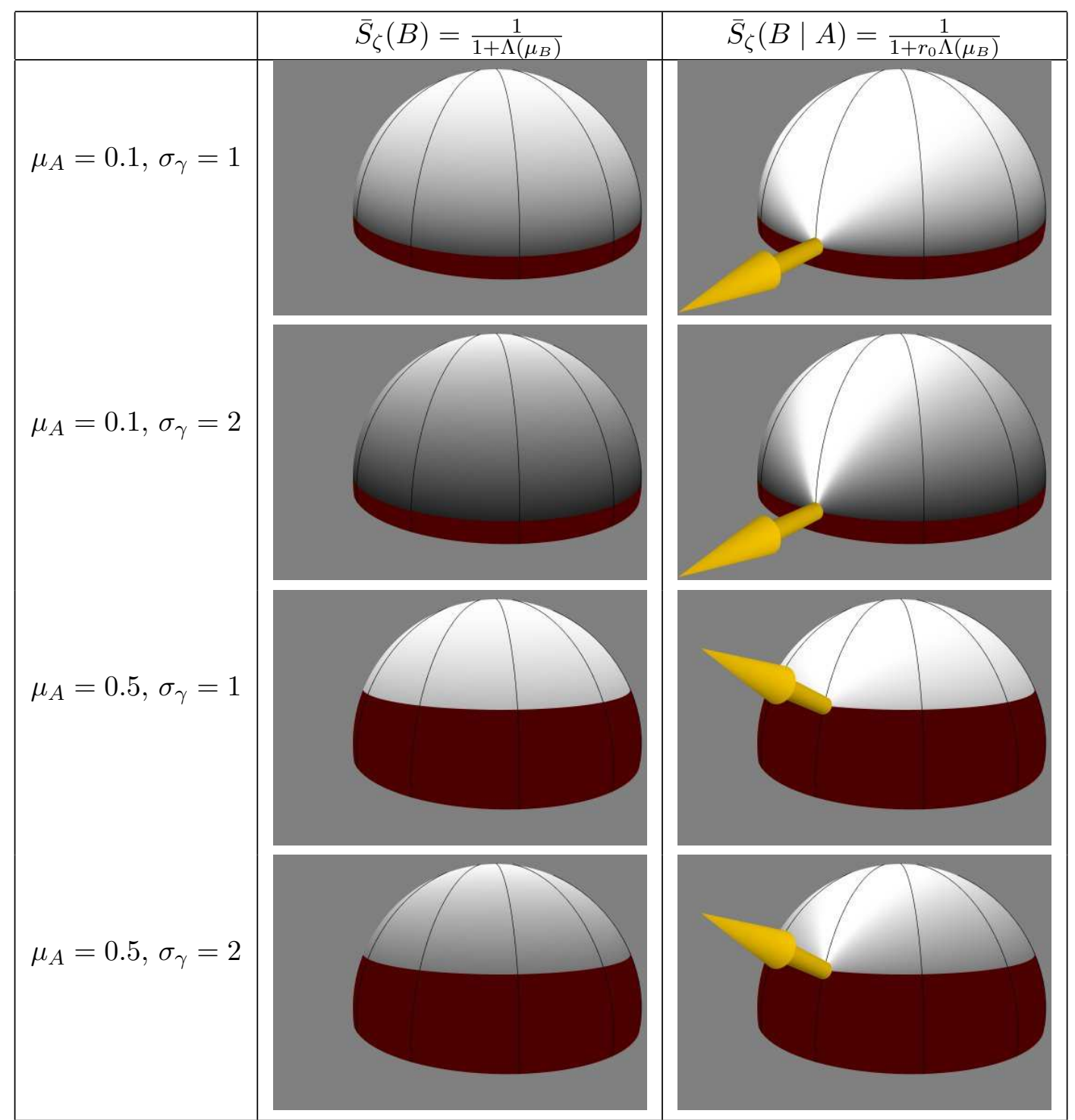

Figure 7. Comparison between the height-averaged illumination function $\bar{S}_{\zeta}(B)$ and the conditional height-averaged illumination function $\bar{S}_{\zeta}(B \mid A)$ for several values of $\mu_{A}$ and $\sigma_{\gamma}$. The probabilities are displayed in gray-scale level (white $=1$ and black $=0$ ). The points on the hemisphere are associated to the direction $B$ and the arrow points to the direction $A$. In the red zone $\mu_{B}<\mu_{A}$, our model does not apply.

integrate to compute the conditional height-averaged expression

$$
\begin{aligned}
\bar{S}_{\zeta}(B \mid A) & =\int_{-\infty}^{\infty} P_{\zeta}\left(\zeta_{0}\right)^{r_{0} \Lambda\left(\mu_{B}\right)} p_{\zeta}\left(\zeta_{0}\right) d \zeta_{0} \\
& =\frac{1}{1+r_{0} \Lambda\left(\mu_{B}\right)}
\end{aligned}
$$

Figure 7 illustrates the effect of the factor $r_{0}$ in the conditional height-averaged illumination function for several values of $\mu_{A}$ and $\sigma_{\gamma}$.

\subsubsection{Conclusion}

For a 2-D surface and observation directions $A$ and $B$ such that $\mu_{A}<\mu_{B}$, the bistatic illumination function is expressed as

$$
S(F, A \cap B)=S_{\gamma}\left(\gamma_{0 x}, \gamma_{0 y}, A \cap B\right) S_{\zeta}\left(\zeta_{0}, A\right) S_{\zeta}\left(\zeta_{0}, B \mid A\right)
$$


The probability $S_{\zeta}\left(\zeta_{0}, A\right)=P_{\zeta}\left(\zeta_{0}\right)^{\Lambda\left(\mu_{A}\right)}$ is expressed from Eq. (5) $\left(\theta \rightarrow \theta_{A}\right)$ and the probability $S_{\zeta}\left(\zeta_{0}, B \mid A\right)=P_{\zeta}\left(\zeta_{0}\right)^{r_{0} \Lambda\left(\mu_{B}\right)}$. In addition, the probability $S_{\gamma}\left(\gamma_{0 x}, \gamma_{0 y}, A \cap B\right)$ is expressed from Eq. (10).

The slope-averaging is discussed in subsection 2.3.1, and the slope-averaged expression is

$$
\bar{S}\left(\zeta_{0}, A \cap B\right)=\bar{S}_{\gamma}(A \cap B) S_{\zeta}\left(\zeta_{0}, A\right) S_{\zeta}\left(\zeta_{0}, B \mid A\right)
$$

Since $r_{0}$ does not depend on the height, we can easily integrate to compute the height-averaged expression

$$
\begin{aligned}
\bar{S}\left(\gamma_{0 x}, \gamma_{0 y}, A \cap B\right) & =S_{\gamma}\left(\gamma_{0 x}, \gamma_{0 y}, A \cap B\right) \int_{-\infty}^{\infty} S_{\zeta}\left(\zeta_{0}, A\right) S_{\zeta}\left(\zeta_{0}, B \mid A\right) p_{\zeta}\left(\zeta_{0}\right) d \zeta_{0} \\
& =S_{\gamma}\left(\gamma_{0 x}, \gamma_{0 y}, A \cap B\right) \int_{-\infty}^{\infty} P_{\zeta}\left(\zeta_{0}\right)^{\Lambda\left(\mu_{A}\right)+r_{0} \Lambda\left(\mu_{B}\right)} p_{\zeta}\left(\zeta_{0}\right) d \zeta_{0} \\
& =\frac{S_{\gamma}\left(\gamma_{0 x}, \gamma_{0 y}, A \cap B\right)}{1+\Lambda\left(\mu_{A}\right)+r_{0} \Lambda\left(\mu_{B}\right)}
\end{aligned}
$$

The height- and slope-averaged expression is

$$
\overline{\bar{S}}(A \cap B)=\frac{\bar{S}_{\gamma}(A \cap B)}{1+\Lambda\left(\mu_{A}\right)+r_{0} \Lambda\left(\mu_{B}\right)}
$$

\section{Results}

Figure 8 presents comparisons of the surface average bistatic illumination function $\overline{\bar{S}}(A \cap B)$ in function of the azimuthal difference $\phi$ between $A$ and $B$. We compare the following results:

- Monte Carlo: we use 3000 samples per configuration to integrate the bistatic illumination on an artificially-generated Gaussian surface with Gaussian autocorrelation function.

- Bistatic 1-D is the result obtained when $A$ and $B$ are in the same plane. In this case, $\overline{\bar{S}}(A \cap B)=\overline{\bar{S}}(A)$. This result is constant in Figure 8, because it assumes $\phi=0$.

- Bistatic 2-D uncorrelated is the separable product $\overline{\bar{S}}(A \cap B)=\overline{\bar{S}}(A) \overline{\bar{S}}(B)$. This result is constant in Figure 8 because it is not a function of the variable $\phi$.

- Bourlier's model takes account of the slope correlation, but neglects azimuthal correlation in the height illumination functions. He evaluates

$$
\overline{\bar{S}}(A \cup B)=\bar{S}_{\gamma}(A \cap B) \bar{S}_{\zeta}(A \cap B)=\frac{\bar{S}_{\gamma}(A \cap B)}{1+\Lambda\left(\mu_{A}\right)+\Lambda\left(\mu_{B}\right)}
$$

- Our model is Bourlier's model where $\Lambda\left(\mu_{B}\right)$ is multiplied with our analytic approximation of the correction coefficient $r_{0}$

$$
\overline{\bar{S}}(A \cup B)=\bar{S}_{\gamma}(A \cap B) \bar{S}_{\zeta}(A \cap B)=\frac{\bar{S}_{\gamma}(A \cap B)}{1+\Lambda\left(\mu_{A}\right)+r_{0} \Lambda\left(\mu_{B}\right)}
$$

The plot of the Monte-Carlo simulation show that the correlation effect is important when $\phi$ is small and when the observation angle $\theta$ is high (grazing angles). 


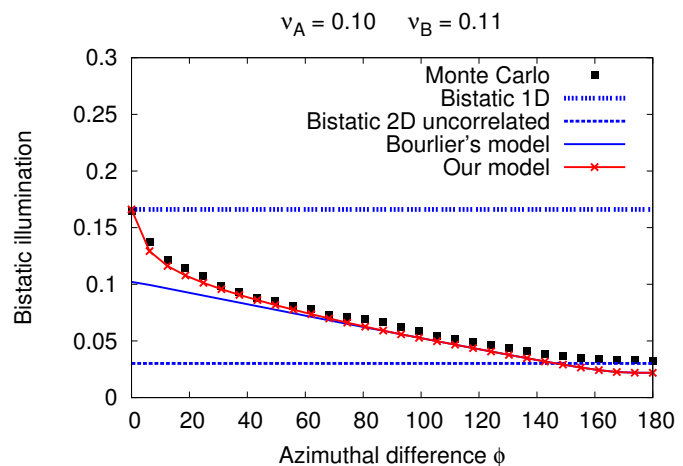

(a)

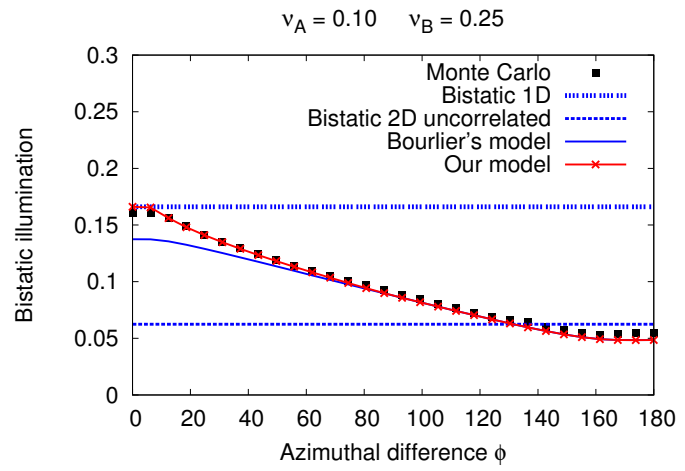

(c)

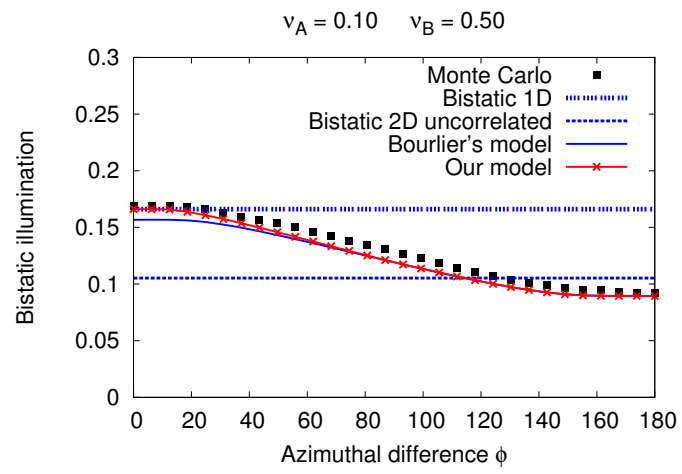

(e)

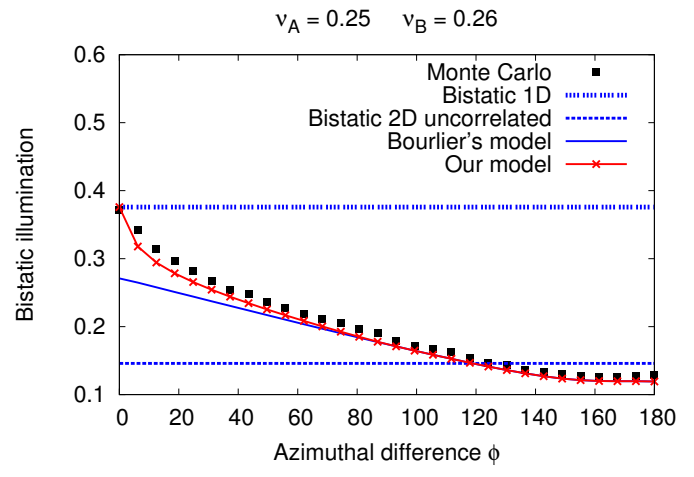

(b)

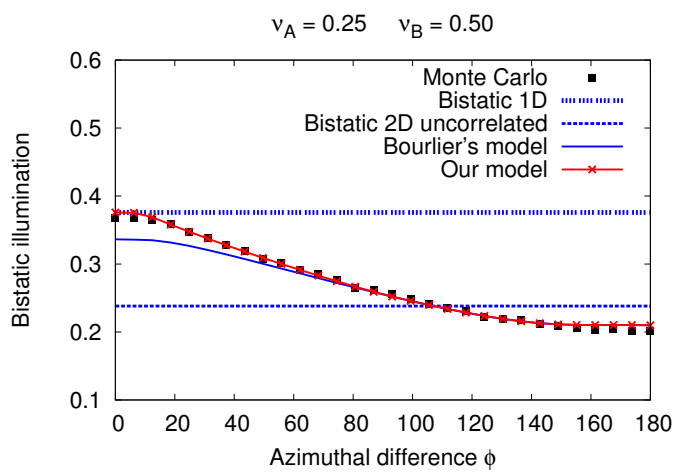

(d)

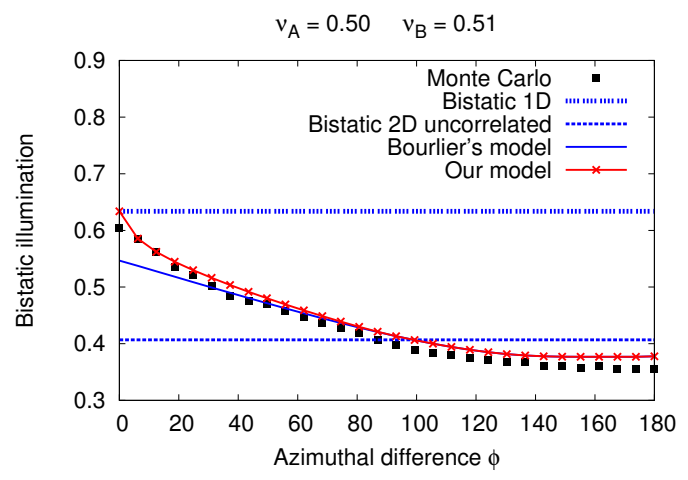

(f)

Figure 8. Comparison of Monte Carlo results with different bistatic illumination functions. The parameters of the configuration are the normalized slopes $\nu=\mu / \sqrt{2} \sigma_{\gamma}$.

Intuitively, the less the point is visible, the more the correlation effect becomes significant.

\section{Conclusion}

In this paper, we have shown that the correlation between the transmitter and receiver illumination functions is an important effect and should be taken in account. We have proposed a model based on the Smith formulation in which we modified the formula by using the information of the first ray to modify the surface statistics in the computation of the second ray. We have shown that the information 
provided by the first ray can be modelled in the final formulae by introducing a factor $r_{0}$ for which we proposed a simple and accurate analytical approximation.

We used the hypothesis of independent heights and slopes to model the information provided by a measure point on the surface. Bourlier et al. [4] showed that this correlation is not important in monostatic configurations, but it may be more significant in the bistatic case. We also limited our approach to isotropic Gaussian surfaces. This work could be extended to other kinds of surfaces, since illumination functions have been studied on more general surface statistics $[3,5]$.

This work is an attempt to step in a difficult problem not addressed in current previous work. While our derivation has some limitations, we hope that this lead will motivate and inspire future work on this topic. 


\section{Appendix A. Matlab code for $r_{0}$}

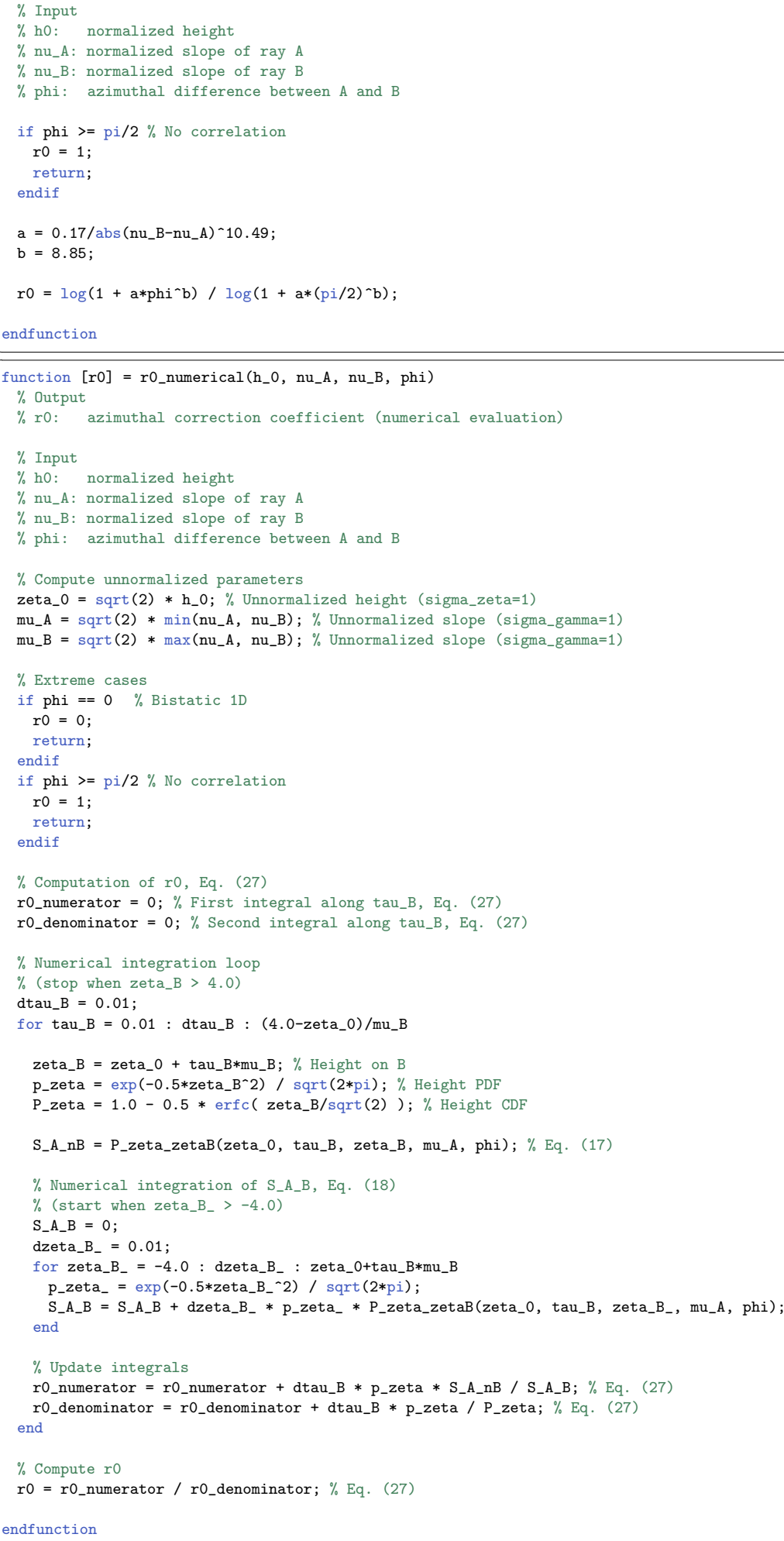




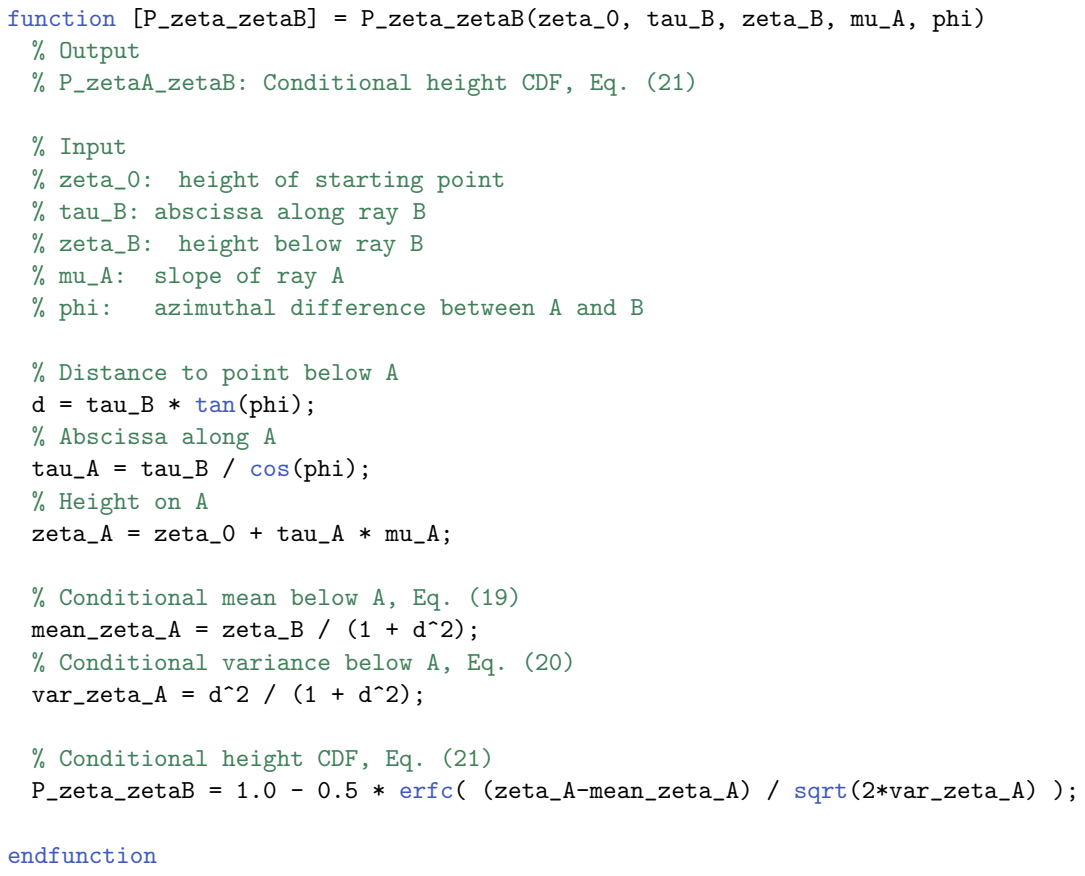

\section{References}

[1] R.J. Wagner, Shadowing of Randomly Rough Surfaces, The Journal of the Acoustical Society of America 41 (1967), pp. 138-147.

[2] B. Smith, Geometrical shadowing of a random rough surface, IEEE Transactions on Antennas and Propagation 15 (1967), pp. 668-671.

[3] G. Brown, Shadowing by non-Gaussian random surfaces, IEEE Transactions on Antennas and Propagation 28 (1980), pp. $788-790$.

[4] C. Bourlier, J. Saillard, and G. Berginc, Effect of correlation between shadowing and shadowed points on the Wagner and Smith monostatic one-dimensional shadowing functions, IEEE Transactions on Antennas and Propagation 48 (2000), pp. 437-446.

[5] C. Bourlier, G. Berginc, and J. Saillard, One- and two-dimensional shadowing functions for any height and slope stationary uncorrelated surface in the monostatic and bistatic configurations, IEEE Transactions on Antennas and Propagation 50 (2002), pp. 312 -324.

[6] Bourlier and G. Berginc, Shadowing function with single reflection from anisotropic Gaussian rough surface. Application to Gaussian, Lorentzian and sea correlations, Waves in Random and Complex Media 13 (2003), pp. 27-58.

[7] Y. Zhu Multisensor Decision and Estimation Fusion, Springer, 2003. 